\title{
Increased cerebrovascular reactivity in selected brain regions after extracranial-intracranial bypass improves the speed and accuracy of visual cancellation in patients with severe steno-occlusive disease: a preliminary study
}

\author{
Koji Shimonaga ${ }^{1}$ - Seiji Hama ${ }^{2,3,4}\left(\right.$ Akira Furui $^{5} \cdot$ Akiko Yanagawa $^{2,3,4} \cdot$ Akihiko Kandori $^{6} \cdot$ Hirokazu Atsumori $^{6}$. \\ Shigeto Yamawaki ${ }^{4} \cdot$ Toshinori Matsushige $^{1} \cdot$ Toshio Tsuji $^{5}$
}

Received: 16 June 2021 / Revised: 24 October 2021 / Accepted: 13 December 2021 / Published online: 30 January 2022

(c) The Author(s) 2021

\begin{abstract}
The effect of the change in cerebrovascular reactivity (CVR) in each brain area on cognitive function after extracranialintracranial bypass (EC-IC bypass) was examined. Eighteen patients who underwent EC-IC bypass for severe unilateral steno-occlusive disease were included. Single-photon emission CT (SPECT) for evaluating CVR and the visual cancellation (VC) task were performed before and after surgery. The accuracy of VC was expressed by the arithmetic mean of the agematched correct answer rate and the accurate answer rate, and the averages of the time (time score) and accuracy (accuracy score) of the four VC subtests were used. The speed of VC tended to be slower, whereas accuracy was maintained before surgery. The EC-IC bypass improved CVR mainly in the cerebral hemisphere on the surgical side. On bivariate analysis, when CVR increased post-operatively, accuracy improved on both surgical sides, but the time score was faster on the left and slower on the right surgical side. Stepwise multiple regression analysis showed that the number of the brain regions associated with the time score was 5 and that associated with the accuracy score was 4 . In the hemodynamically ischemic brain, processing speed might be adjusted so that accuracy would be maintained based on the speed-accuracy trade-off mechanism that may become engaged separately in the left and right cerebral hemispheres when performing VC. When considering the treatment for hemodynamic ischemia, the relationship between CVR change and the speed-accuracy trade-off in each brain region should be considered.
\end{abstract}

Keywords Extracranial-intracranial bypass $\cdot$ Cognitive function $\cdot$ Speed-accuracy trade-off $\cdot$ Severe steno-occlusive disease $\cdot$ Visual cancellation

Seiji Hama

shama@hiroshima-u.ac.jp

1 Department of Neurosurgery and Interventional Neuroradiology, Hiroshima City Asa Citizens Hospital, Hiroshima 731-0293, Japan

2 Department of Neurosurgery, Graduate School of Biomedical and Health Sciences, Hiroshima University, 1-2-3 Kasumi, Minami-ku, Hiroshima 734 8551, Japan

3 Department of Rehabilitation, Hibino Hospital, Hiroshima 731-3164, Japan

4 Center for Brain, Mind and KANSEI Sciences Research, Hiroshima University, Hiroshima 734-8551, Japan

5 Graduate School of Advanced Science and Engineering, Hiroshima University, Hiroshima 739-8527, Japan

6 Center for Exploratory Research, Research and Development Group, Hitachi. Ltd, Tokyo 185-8601, Japan

\section{Introduction}

Symptomatic major cerebral arterial occlusion or stenosis makes the status of cerebral hemodynamics progress from stage 0 (normal cerebral hemodynamics) to stage 1 hemodynamic ischemia (stage 1) (autoregulatory vasodilation to keep cerebral blood flow (CBF) normal) and then stage 2 hemodynamic ischemia (stage 2) (CBF cannot be maintained, and brain tissues increase the oxygen extraction fraction (OEF)) as the stenosis progresses, and cerebral infarction becomes inevitable before long [1-4]. To prevent progression from stage 2 to cerebral infarction, extracranial-intracranial (EC-IC) bypass surgery has been considered [5-7]. However, the selection criteria for medical treatment or surgical treatment have been controversial 
until now $[4,8]$. One of the key issues lies in how to evaluate stage 2 , especially the signs of progression to cerebral infarction.

Although positron emission tomography (PET) has the advantage of being able to diagnose stage 2 because OEF can be measured [4], its clinical availability is limited by its high cost and complexity [2]. Instead, single-photon emission CT (SPECT)-measured cerebrovascular reactivity (CVR), which is defined as the change in CBF in response to a vasoactive stimulus, has been used [2, 3, 9]. According to a Japanese Extracranial-Intracranial Bypass Trial (JET) study, if rest CBF was less than $80 \%$ of the normal value and CVR was less than 10\%, EC-IC bypass surgery was more effective in preventing cerebral infarction than drug therapy $[5,6]$.

Cerebral hypoperfusion induced by steno-occlusive diseases has been thought to be associated with cognitive decline [10-12]. If so, the status of cerebral hemodynamics (especially stage 2) may be estimated using cognitive function tests. Some previous reports suggested that EC-IC bypass improves cognitive function in patients with stenoocclusive disease $[11,12]$. However, these previous studies have some limitations, and the effect of EC-IC bypass on cognitive function has remained controversial [12, 13].

CVR shows the ability of the cerebral vascular bed (regulation of vasodilatation and collateral blood flow) to maintain a balanced distribution of blood flow and varies by brain region [3]. In addition, cognitive function is classified into many types, each of which is associated with a specific brain region $[14,15]$. Thus, the association between CVR and cognitive function before and after EC-IC bypass should be examined after dividing the brain into regions.

We previously demonstrated that right hemisphere damage led to a delay in cognitive processing speed after stroke [16]. Skurvydas and colleagues studied hand and leg movement control in stroke patients in the right cerebral hemisphere and showed that they perform impulsive movements at the expense of inaccurate movements [17]. This is a wellknown theory in the kinesiology or neuroscience, but it is little known in the stroke or neurosurgical field, called the "speed-accuracy trade-off," which implies that faster movement can be performed at the cost of reduced accuracy and vice versa [18]. The speed-accuracy trade-off mechanism might be associated with cognitive function in the process from hemodynamic ischemia (especially stage 2 ) to cerebral infarction.

The present study was a preliminary test of the hypothesis that "regional CVR changes" and "speed-accuracy trade-off theory" can be used to clarify the relationship between cognitive function and CVR, to clarify the cognitive dysfunction that accompanies the progression from hemodynamic ischemia to cerebral infarction.

\section{Material and methods}

\section{Patients}

This retrospective study was conducted as a preliminary study after approval by the Research Ethics Committee of Hiroshima City Asa Citizens Hospital and Hiroshima University (E-466-3, E-1554-2). Written, informed consent was obtained from all participants. In this study, 18 consecutive patients who underwent EC-IC bypass for severe unilateral steno-occlusive disease of the intracranial internal carotid artery (ICA) or middle cerebral artery (MCA) at Asa Citizens Hospital from September 2017 to April 2020 were reviewed. All patients experienced transient ischemic attacks (TIAs) or non-disabling strokes within 6 months derived from the hemisphere ipsilateral to the lesion.

CBF and CVR were estimated by SPECT. The regional $\mathrm{CBF}(\mathrm{rCBF})$ of the ipsilateral cerebral cortex region was compared by the value divided by the $\mathrm{rCBF}$ of the ipsilateral cerebellar cortex region [19]. Then, according to the JET study, EC-IC bypass was indicated for $\mathrm{rCBF}$ reduction of less than $80 \%$ and regional CVR (rCVR) reduction of less than $10 \%[5,6]$. In all cases, no stenotic lesions causing decreased cerebellar blood flow were observed in the vertebrobasilar arteries.

The inclusion criteria for this study were patients with available pre-operative and post-operative (3-6 months or later after surgery) CVR studies. Exclusion criteria were as follows: (1) allergy to contrast media; (2) renal dysfunction (estimated glomerular filtration rate $<30 \mathrm{ml} / \mathrm{min} / 1.73$ $\mathrm{m}^{2}$ ); or (3) medical illness, physical disability, or aphasia (modified Rankin scale score $\geq 3$ ) precluding the VC task. Eighteen patients were selected on the basis of the inclusion criteria ( 5 women; mean age at the time of bypass 68.1 years). The etiology included atherosclerosis $(n=18)$ (Table 1).

\section{Assessment of cognitive functions (time and accuracy score)}

We routinely perform cognitive function tests (mini-mental state examination, trail making test, and the Clinical Assessment for Attention (CAT)) within 3 days of SPECT before and 3-6 months after surgery. Of these cognitive tests, the duration and accuracy of visual cancellation (VC), position Stroop test, and Continuous Performance Test could be evaluated at the same time, but only the VC was performed in all 18 cases. Thus, the results of $\mathrm{VC}$ were used for the following analysis. VC consisted of four kinds of subtests (Kana, Triangle, Symbol, Number) 
Table 1 Patients' demographics

\begin{tabular}{lllll}
\hline & All & \multicolumn{3}{l}{ Side of EC-IC bypass } \\
\cline { 3 - 4 } & & Lt $(n=11)$ & Rt $(n=7)$ & $p$ \\
\hline Age \pm SE $(y)$ & $68.111 \pm 9.934$ & $67.364 \pm 10.043$ & $69.286 \pm 10.436$ & 0.751 \\
Sex; male $(\%)$ & $13(72.22 \%)$ & $8(72.73 \%)$ & $5(71.43 \%)$ & 0.952 \\
Time score & & & & \\
Pre-op \pm SE & $1.801 \pm 1.202$ & $1.675 \pm 0.643$ & $2.001 \pm 1.825$ & 0.684 \\
Post-op \pm SE & $1.684 \pm 1.427$ & $1.502 \pm 0.575$ & $1.970 \pm 2.250$ & 0.497 \\
Change \pm SE & $-0.117 \pm 0.436$ & $-0.172 \pm 0.383$ & $-0.031 \pm 0.530$ & 0.964 \\
Accuracy score & & & & \\
Pre-op \pm SE & $0.987 \pm 0.027$ & $0.982 \pm 0.0280$ & $0.994 \pm 0.024$ & 0.16 \\
Post-op \pm SE & $0.991 \pm 0.014$ & $0.988 \pm 0.016$ & $0.996 \pm 0.009$ & 0.222 \\
Change \pm SE & $0.005 \pm 0.025$ & $0.006 \pm 0.029$ & $0.002 \pm 0.016$ & 0.751 \\
\hline
\end{tabular}

The Mann-Whitney test was used to compare continuous variables, and the $\chi^{2}$ test was used to compare categorical variables

$S E$ standard error

included in CAT, which is a standardized test for attention deficit, that were used as previously described [15]. Participants used a pencil to cross out a target stimulus dispersed within rows of randomly placed interfering stimuli displayed on a sheet. These tasks were scored as speed (completion time) and accuracy. Accuracy was based on the ratio of the number of correct answers to the total number of items (\% correct answers) or the number of accurate answers compared to the number of total responses (both correct and incorrect responses) (\% accurate answer).

Fig. 1 Box plots and dot plots of differences in the average age-matched completion time (A) and \% correct answers, $\%$ accurate answers, and the accuracy score (B) between pre(white) and post- (gray) extracranial-intracranial (EC-IC) bypass of the 4 visual cancellation (VC) subtests. Box plots show medians, quartiles, and 10th and 90th percentiles. The dotted line shows the average. Dash-dot-dash line shows the average pre- and post-operative completion time or \% correct, $\%$ accurate, and accuracy score of $4 \mathrm{VC}$ subtests. Score $=1$ indicates the age-matched average
(A)

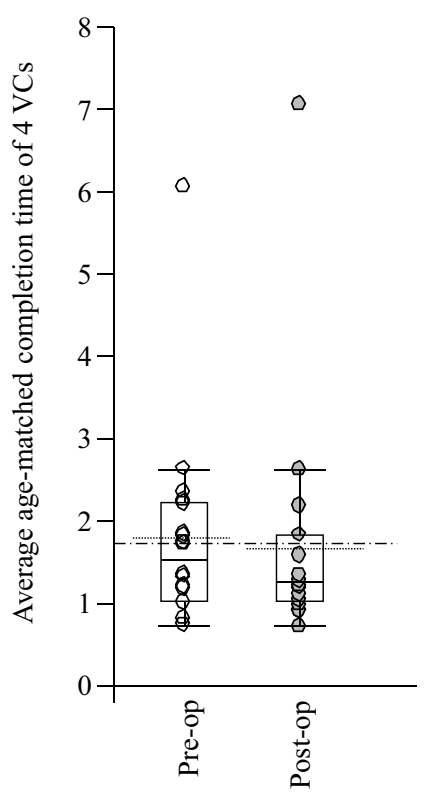

It is known that the scores of the VC depend on age. To correct by age, age-matched values were calculated as (VC score) / (age-specific mean VC value); the lower the age matched $\%$ correct answer and $\%$ accurate answer, the greater the attentional disturbance, and the higher the agematched completion time (the slower the speed), the greater the attentional disturbance.

The accuracy of each VC subtest was evaluated by (age matched $\%$ correct answer + age-matched $\%$ accurate answer) $/ 2$, and the following analysis was performed with

(B)

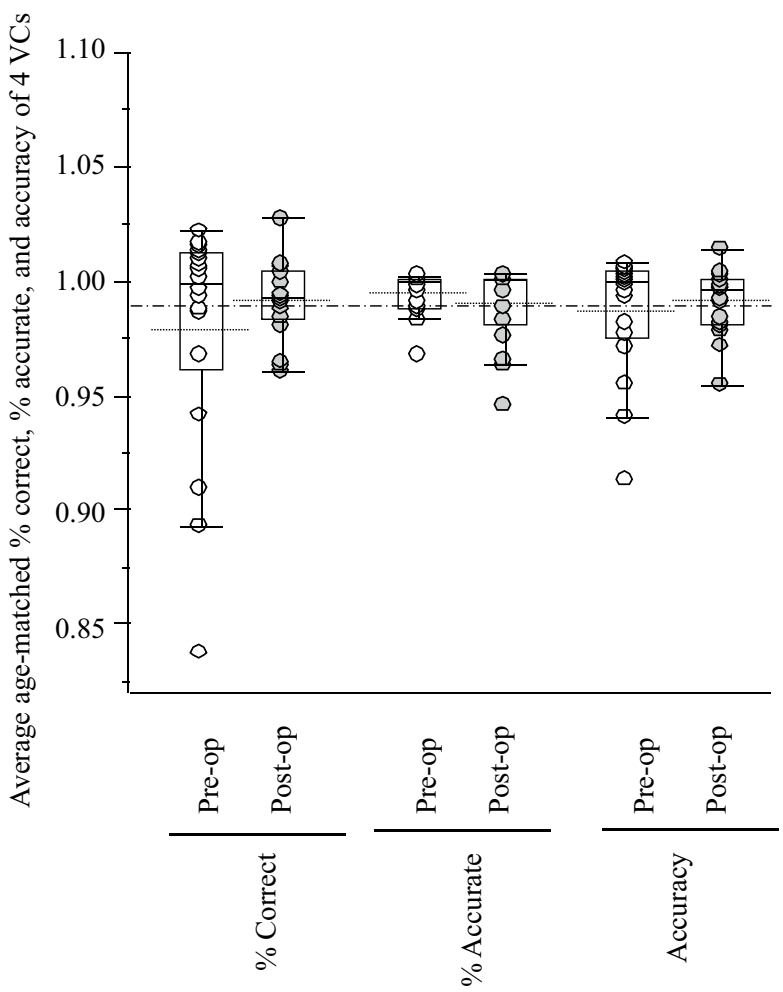


(A) Lt bypass $(\mathrm{n}=11)$

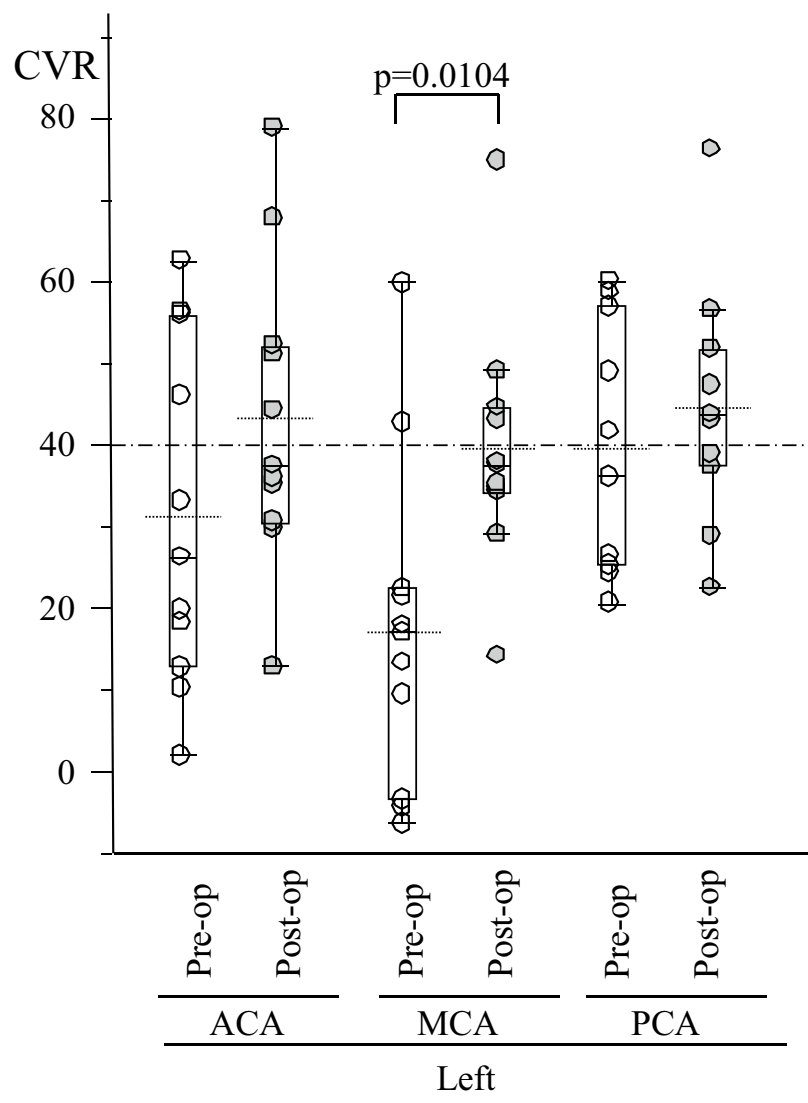

(B) Rt bypass $(n=7)$

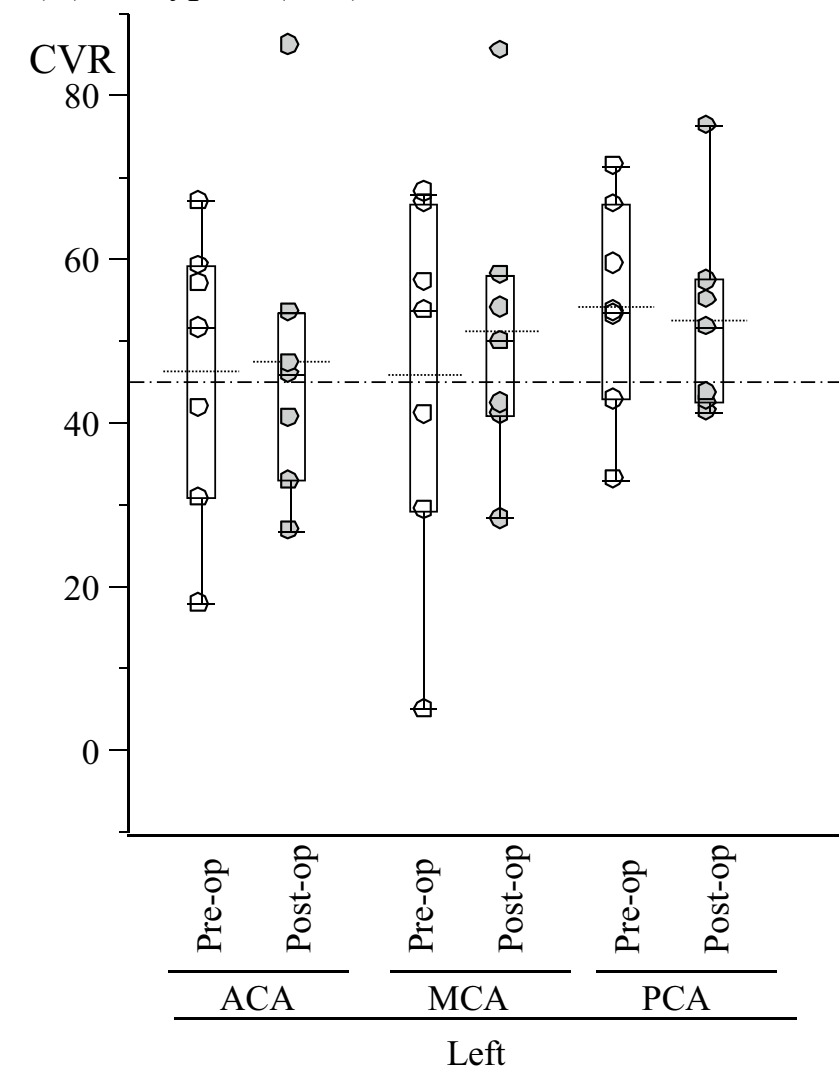

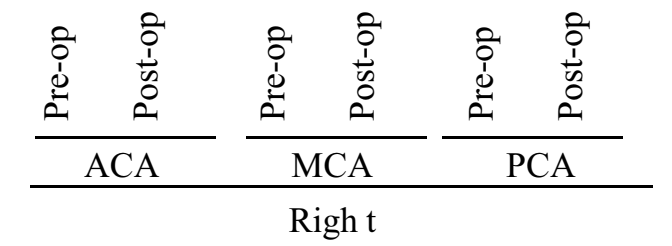

- $\quad \mathrm{p}=0.0049$

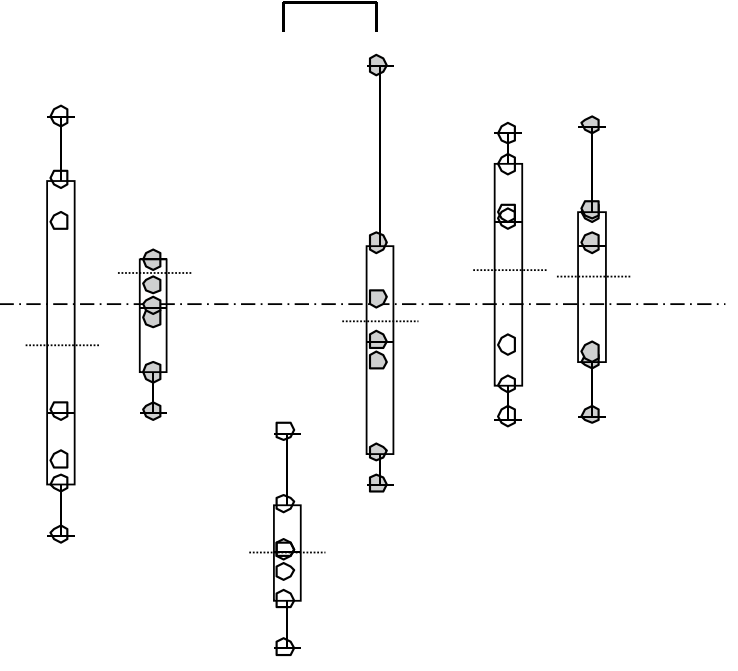


4Fig. 2 Box plots and dot plots of differences in CVR change in right or left ACA, MCA, and PCA territories between pre- (white) and post- (gray) EC-IC bypass (vertical lines indicate standard deviation) classified by treatment side (A; left, B; right). Box plots show medians, quartiles, and 10th and 90th percentiles. The dotted line shows the average. Dash-dot-dash line shows the average pre- and post-operative CVR. The $p$ value was obtained using the Mann-Whitney $U$ test

the average value of the four accuracies (accuracy score). In addition, the following analysis was performed using the average value of the age-matched completion times of four VCs (time score). A score of 1 of both the accuracy score and the time score indicates the age-corrected average value.

\section{Measurement and analysis of CBF and CVR using SPECT}

All subjects in this study received a 222-MBq dose of $N$-isopropyl- ${ }^{123}$ I- $p$-iodoamphetamine (IMP) intravenously. Siemens e.cam (Siemens Medical Solutions, Erlangen, Germany) was used in 18 patients to acquire the projection data in a continuous mode at $150 \mathrm{~s} /$ cycle $\left(180^{\circ}\right.$ rotation of dual heads) for 2 cycles, repeated 6 times. In both machines, lowenergy high-resolution collimators were used, with a matrix size of $128 \times 128$ and an energy window of $159 \mathrm{keV} \pm 15 \%$. After the data were obtained, a three-dimensional stereotactic surface profile program (3D-SSP, Nihon Medi-Physics, Tokyo, Japan) was used to spatially normalize the local distribution. In brief, the coordinate data were converted to the normal brain based on the Talairach brain atlas, classified into segments, and the $\mathrm{Z}$ score was calculated by comparing with a normal database using SEE (stereotactic extraction estimation) analysis, and the CBF and CVR in each brain region were evaluated [20].

In this study, the rCBF values were obtained by SPECT with the graph plot method that uses IMP, which does not require arterial blood sampling [21]. Thus, arterial sampling was not performed during SPECT.

Scans for rCBF were performed just before and $10 \mathrm{~min}$ after injection of $1.0 \mathrm{~g}$ of acetazolamide. Regional cerebrovascular reactivity (rCVR) was calculated as follows:
$\operatorname{rCVR}(\%)=[($ acetazolamide challenge $\mathrm{rCBF}-$ resting $\mathrm{rCBF})$ / resting $\mathrm{rCBF}] \times 100$. The change in CVR (post-operative CVR - pre-operative CVR) was calculated.

The CVR was divided into three areas (ACA, MCA, and PCA) or the 31 supratentorial areas of 50 regions of level 3 of the anatomical classification based on the Talairach Daemon database on each side (supplemental Table 1) [20].

\section{Operative procedure}

Under general anesthesia, with continued antiplatelet medication perioperatively, a skin incision was made just over the superficial temporal artery (STA) frontal branch or parietal branch. Under microscopy, meticulous STA dissection was conducted. The skin incision was then extended toward the forehead, and a skin flap was reflected. The frontal branch of the STA was dissected. After craniotomy, an STA-MCA single or double anastomosis was performed between each STA branch and the recipient M4 (cortical MCA branch). Successful bypass was confirmed by microvascular Doppler evaluation. EC-IC bypass in this study included only the direct procedure and was not combined with indirect bypass procedures.

\section{Statistical analysis}

To compare differences between two groups, Fisher's exact test was used for categorical variables, and the Mann-Whitney $U$-test was used for quantitative variables. The level of significance was set at $p<0.05$.

To test the correlations between the time or the accuracy score and the CVR in the 31 brain areas on each side (62 in total), bivariate analysis (Spearman's rank correlation coefficient, $\rho$ ) was performed.

Stepwise multiple linear regression analysis was used to estimate the independent effects of predictor variables on the time or accuracy score (forward-backward selection method). These predictor variables were as follows: age, sex, laterality of the operation, and the CVR changes in the brain region with a $p$-value of 0.05 or less in the above bivariate

Table 2 Summary of the relationship between change in the accuracy or time score and each regional CVR change

\begin{tabular}{llllll}
\hline & \multicolumn{2}{l}{ Change in accuracy score } & & \multicolumn{2}{c}{ Change in time score } \\
\cline { 2 - 3 } & Lt EC-IC bypass & Rt EC-IC bypass & & Lt EC-IC bypass & Rt EC-IC bypass \\
\hline Number (\%) with negative $\rho$ (negative correlation) & $4(6.5 \%)$ & $2(3.2 \%)$ & & $49(79.0 \%)$ & $6(9.7 \%)$ \\
Number (\%) with positive $\rho$ (positive correlation) & $58(93.5 \%)$ & $60(96.8 \%)$ & & $13(21.0 \%)$ & $56(90.3 \%)$ \\
Number of $p$-value less than 0.1 & $10(16.1 \%)$ & $13(21.0 \%)$ & & $1(1.6 \%)$ & $19(30.6 \%)$ \\
Number of $p$-value of 0.05 or less & $5(8.1 \%)$ & $6(9.7 \%)$ & & $1(1.6 \%)$ & $9(14.5 \%)$ \\
\hline
\end{tabular}

A summary of bivariate analyses of the time or accuracy scores and CVR changes in each brain region (62 brain regions in total) is shown. The number (\% of 62 total brain regions) of Spearman's rank correlation $(\rho)$ values that are positive (or negative) and the number (\% of 62 total brain regions) that have $p$-values less than 0.1 (or 0.05 or less) are shown separately on the surgical side. See the supplementary Table for all bivariate results 


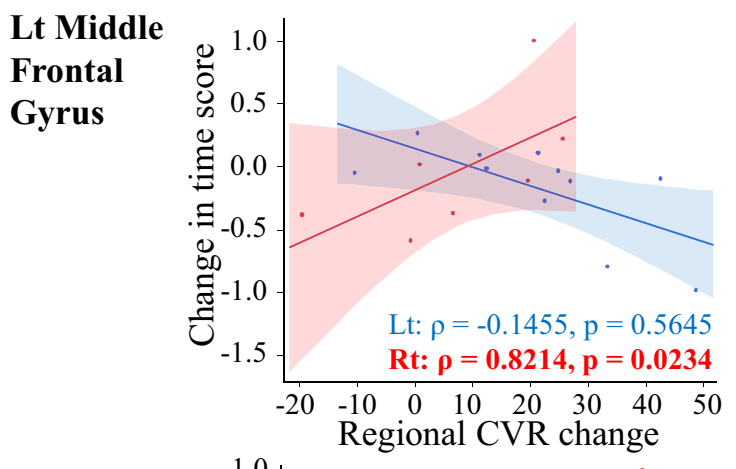

Rt Gyrus Rectus

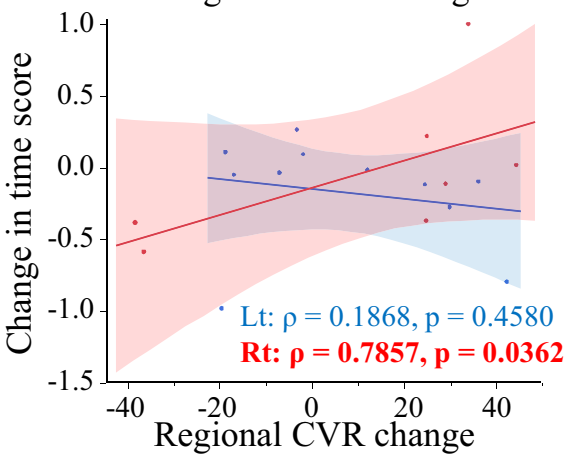

Lt Paracentral Lobule
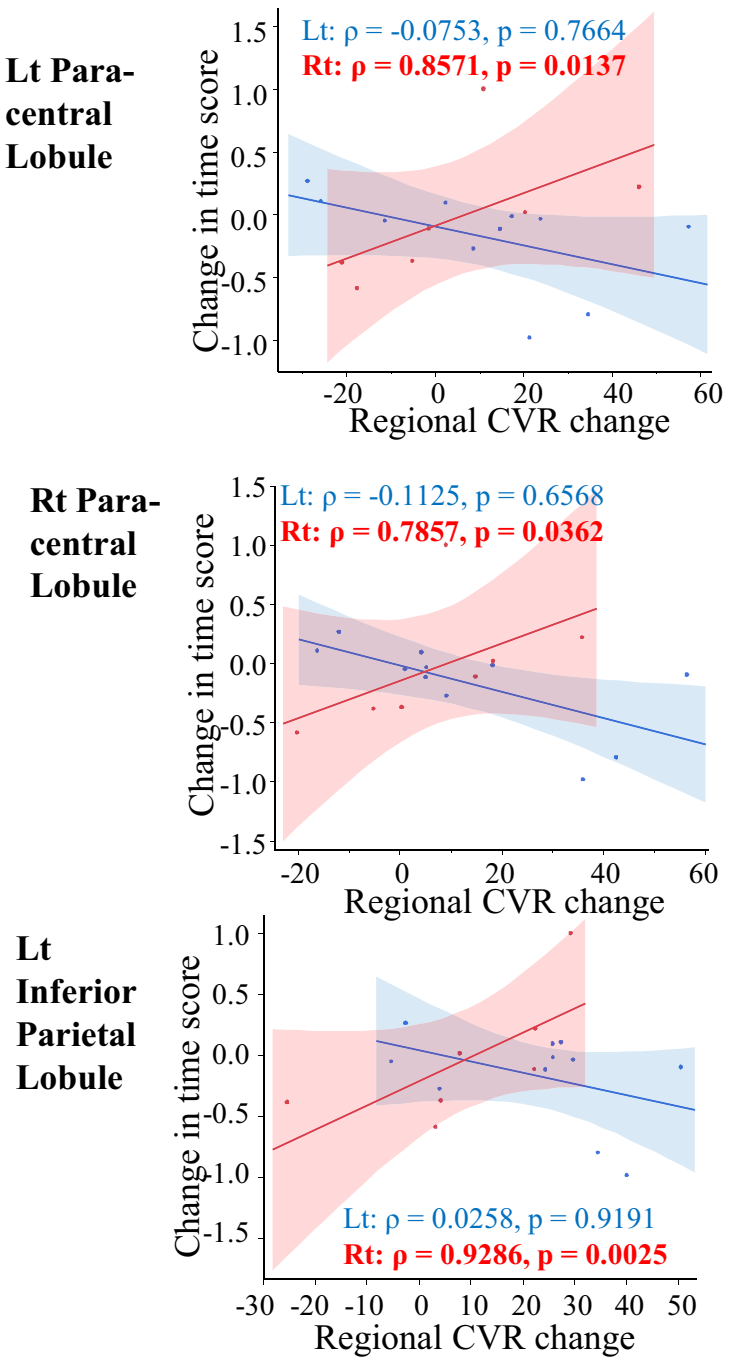
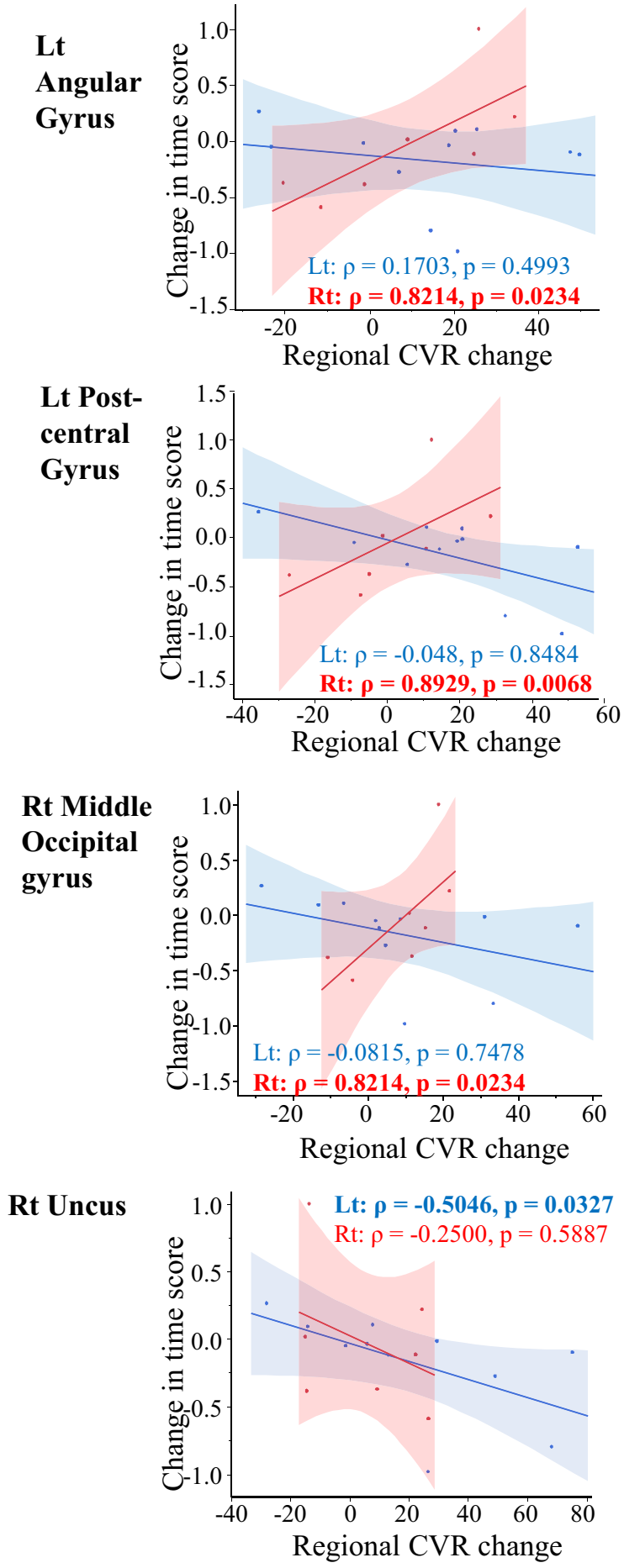

- Lt. bypass $(\mathrm{n}=11)$
- Rt. Bypass $(\mathrm{n}=7)$ 
4Fig. 3 Scatter plots show the correlation between each regional CVR change and the time score change between pre- and post-ECIC bypass. The results showing a $p$-value of 0.05 or less on bivariate analysis are shown (see supplemental Table 1 for all results). The solid lines represent simple linear regression lines. The shaded area indicates the $95 \%$ confidence interval of each regression line. The Spearman rank correlation coefficient $(\rho)$ and $p$ values are presented (bold when the $p$ value is 0.05 or less). All the above are color-coded on the surgery side (blue indicating left and red indicating right EC-IC bypass)

analysis. Logworth was calculated as $-\log _{10}$ ( $p$ value), and higher values were more significant. To assess multicollinearity, the variance inflation factor (VIF) was calculated. A value of 10 was considered to be sufficiently large to indicate multicollinearity [22]. The prediction performance of this model was evaluated through a leave-one-subject-out crossvalidation (i.e., $\mathrm{k}$-fold cross-validation with $k=18$ ).

The values were considered significant at $p<0.05$. All data were analyzed using JMP pro 16.0 (SAS Institute Inc., Cary, NC).

\section{Results}

\section{Baseline characteristics}

A total of 18 consecutive patients were included in this study, all of whom underwent EC-IC bypass (laterality of operation: 7 were right and 11 were left) without adverse events. Magnetic resonance angiography (MRA) confirmed bypass patency in patients 3-6 months after surgery. The basic characteristics are shown in Table 1 . In all cases, the etiology was atherosclerosis, and the dominant hand was the right hand. There were no significant differences in age, sex, time scores, and accuracy scores between the left and right surgical sides.

\section{Visual cancellation task}

The time score (average of age-matched completion time for all four VC tasks) before EC-IC bypass was widely distributed in the range greater than 1, suggesting many cases to be slower than the average completion time (Fig. 1A). The median time score tended to be closer to 1 after EC-IC bypass (completion time became faster), but no significant difference was observed (Fig. 1A).

Compared with the age-matched completion time, the average age-matched $\%$ correct and \% accurate answer of 4 subtests of VC and the accuracy score before EC-IC bypass tended to be distributed in a narrow range close to 1 , and there was no remarkable change after extracranial-intracranial bypass (Fig. 1B, supplemental Table 1).

Therefore, the speed of the visual cancellation task tended to be slower, whereas accuracy was maintained before surgery.

\section{CVR change after extracranial-intracranial bypass}

Figure 2 shows the CVR in bilateral ACA, MCA, and PCA territories before and after left (A) and right side (B) EC-IC bypass. Post-operative CVR in the MCA area on the surgical side was significantly higher than before surgery. The ACA and PCA areas also showed increased CVR after surgery, but not significantly. The EC-IC bypass in this study was considered to have improved CVR mainly in the cerebral hemisphere on the surgical side.

\section{Bivariate analysis}

Spearman's rank correlation coefficients between the time or accuracy score and CVR changes of 62 brain regions were examined on each surgical side. The correlations and $p$-values of all 62 brain regions are shown in supplemental Table 1 and summarized in Table 2.

The correlation between the time score and the CVR change in each brain region was negative on the left surgery side and positive on the right surgery side. The brain region showing a significant CVR change tended to be biased toward the right surgery side (Table 2, Fig. 3).

On the other hand, the correlation between the accuracy score and the CVR change in each brain region was positive on both surgery sides. Moreover, the brain region showing a significant CVR change tended to be similar (Table 2, Fig. 4).

\section{Stepwise multiple regression analysis}

Stepwise multiple linear regression analysis was performed to assess the relationship between the time score or accuracy score and CVR change in 10 or 11 brain regions with the $p$-value of 0.05 or less on the above bivariate analysis.

For the brain regions associated with the time score, a stepwise multiple regression model showed a low predicted value (adjusted $R^{2}$ value $=0.1391$ ), with 5 related regions remaining (Table 3).

For the brain regions associated with the accuracy score, a stepwise multiple regression model showed a low predicted value (adjusted $R^{2}$ value $=0.2533$ ), with 4 related regions remaining (Table 4 ). 

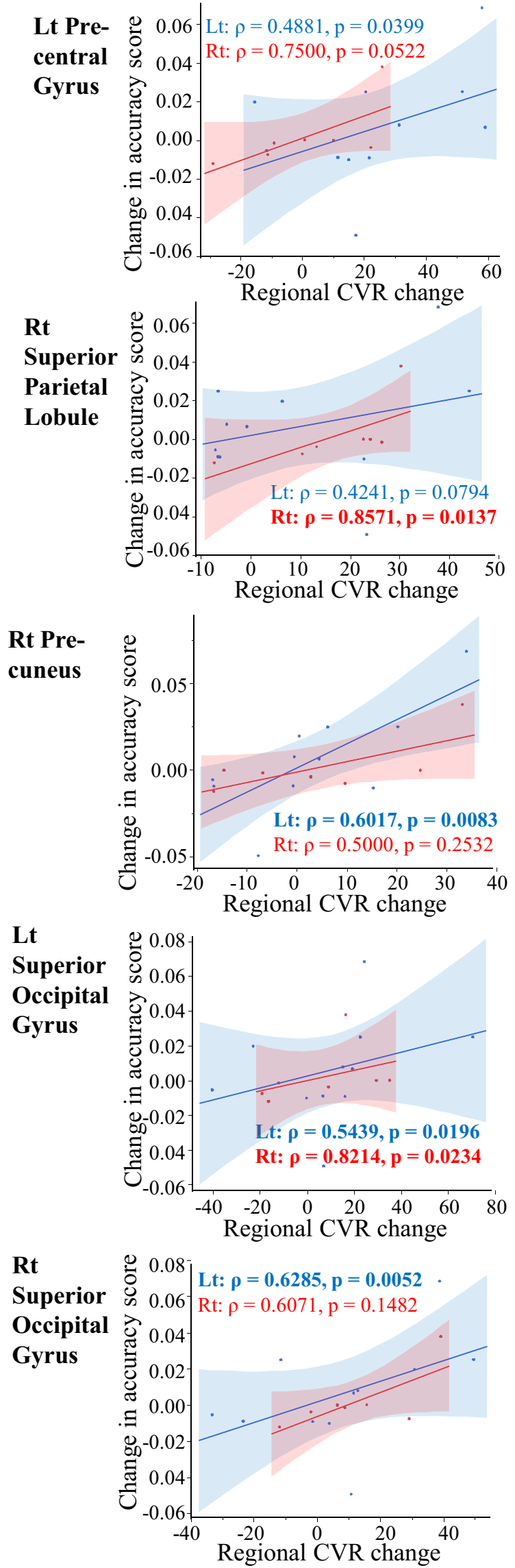

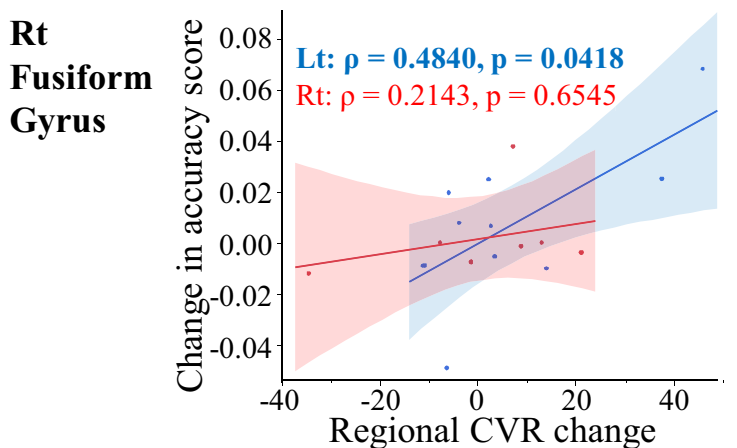

Lt
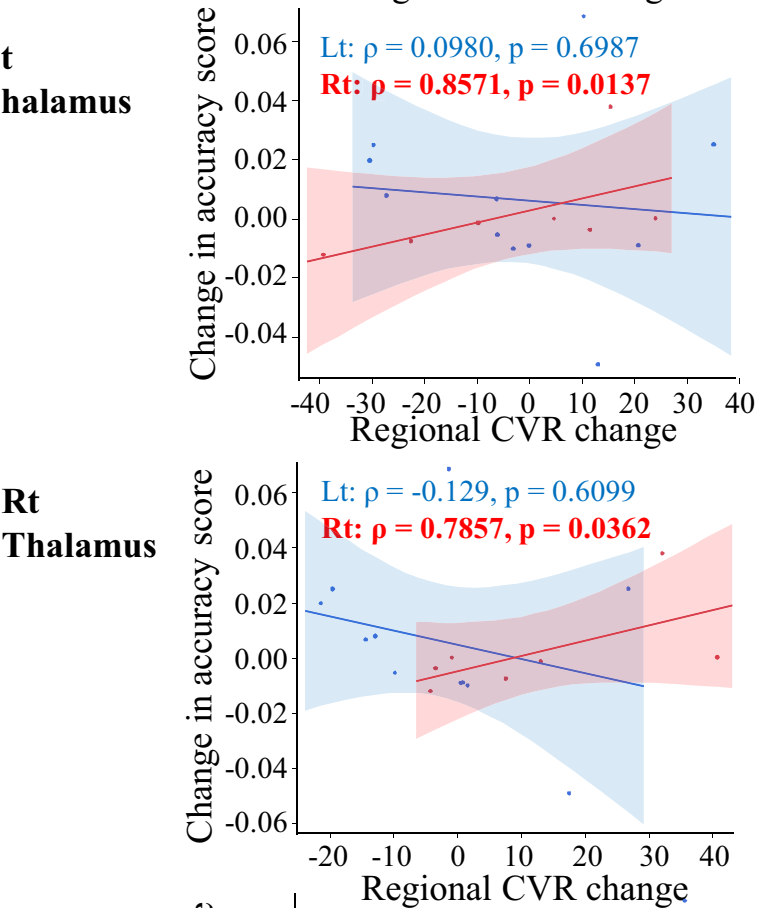

Rt

Cingulate

Gyrus
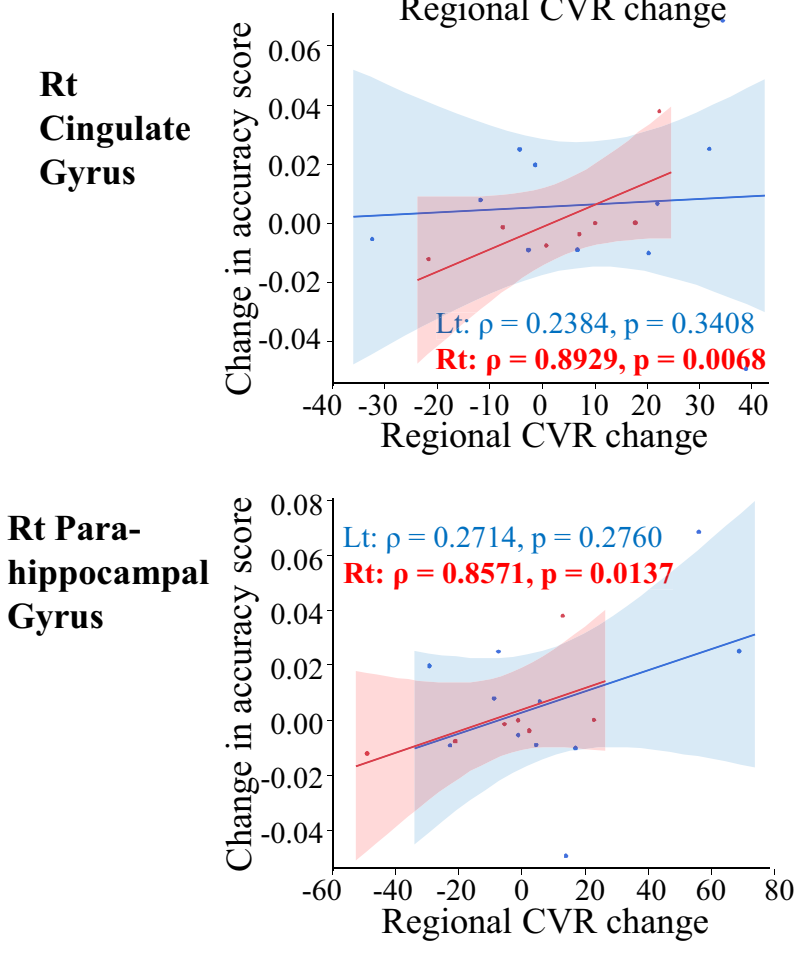
4Fig. 4 Scatter plots show the correlation between each regional CVR change and the accuracy score change between pre- and post-EC-IC bypass. Results showing $p$-values of 0.05 or less on bivariate analysis are shown (see supplemental Table 1 for all results). The solid lines represent simple linear regression lines. The shaded area indicates the $95 \%$ confidence interval of each regression line. The Spearman rank correlation coefficient $(\rho)$ and $p$ values are presented (bold when the $p$ value is 0.05 or less). All the above are color-coded on the surgery side (blue indicating left and red indicating right EC-IC bypass)

\section{Discussion}

In this study, the change in cognitive function after EC-IC bypass surgery was evaluated by dividing the results of VC into time and accuracy scores, and the relationships with the change in CVR were investigated for each brain region. Before EC-IC bypass, the speed of $\mathrm{VC}$ tended to be slower, whereas accuracy was maintained. The EC-IC bypass improved CVR mainly in the cerebral hemisphere (significantly in the MCA territory) on the surgical side. On bivariate analysis, when CVR increased post-operatively, accuracy improved on both the left and right surgical sides, but the time score was faster on the left but slower on the right surgical side. These results may indicate that speed and accuracy may be regulated separately in the left and right cerebral hemispheres when performing VC. This relationship between the time score and the accuracy score has been called the "speed-accuracy trade-off" in the research field of human kinematics (kinesiology) [18].

In the many previous studies of the "speed-accuracy trade-off," inhibition of ongoing thought and action was focused when a mistake was noticed: if the stopping process wins, thought and action are inhibited (slow but more accurate); if the ongoing process wins, thought and action are executed (fast but less accurate) [23]. This inhibition mechanism has been thought to be related to the corticalbasal ganglia circuit; the basal ganglia output an inhibitory signal on the thalamus like a brake and control the speed and accuracy of some action initiated by cerebral cortical activity [24-27]. Moreover, the network systems coordinating motor control and visual signals were thought to be associated with some specific cortical regions, i.e., inferior parietal systems and the temporal lobe [28]. From the theory of the "speed-accuracy trade-off," improvement of CVR after EC-IC bypass may directly increase accuracy or slow down processing speed and indirectly increase accuracy. The mismatch in the relationship between CVR changes and time scores in this study may indicate that the former is on the left and the latter is on the right operative side.

The present results suggest that the left and right cerebral hemispheres regulate speed and accuracy in a coordinated manner. Our previous study that examined the cognitive function related to car driving showed the negative impact of right hemisphere damage on the processing speed in stroke patients, suggesting the importance of preservation of the right hemisphere function for maintaining quality of life [16, 29]. Traditionally, in EC-IC bypass, greater attention has been paid to preservation of the left hemisphere than the right hemisphere. However, given the results of the present study, it is also necessary to consider whether the functions of both the left and right hemispheres are preserved.

\section{Study limitations}

The number of subjects in the present study was small, and statistical analysis issues could have been a problem due to the large number of explanatory variables for the objective variable, the problem of multicollinearity, and overfitting.

Moreover, many previous papers examined the effect of EC-IC bypass on cognitive function with long-term followup (6 months to 2 years or more), but the present study had relatively short follow-up (3 months). In the future, it will be necessary to carry out analyses using data obtained from more cases with longer-term follow-up.

In the present study, CBF measurement was performed using IMP-SPECT and the graph plot method. This method is non-invasive but semi-quantitative, because it does not collect arterial blood [21]. However, the CVR calculated in this study is a method for measuring CBF at rest and after acetazolamide administration in 1 day and is thought to be suitable for comparison between patients in the same institution. Therefore, CVR was used for analysis instead of CBF. To clarify the relationship between cerebral hemodynamics and cognitive function, quantitative CBF measurement using arterial blood sampling or PET measurement will be needed.

The spatial resolution of the CVR images was low, and it was difficult to analyze the small anatomical structures. Thus, further study using a high-resolution method (i.e., MRI) will be needed to clarify the relationship between cognitive function and brain region.

Identification of dominant and non-dominant hemispheres is one of the key factors in brain imaging. In the present study, only hand dominance was examined. Therefore, it will be necessary in the future to identify dominant hemispheres using positron emission tomography (PET) or functional magnetic resonance imaging (fMRI).

The multivariate analysis in this study had low predicted values. The small number of cases may be the cause, but it is also considered that the correlation between the CVR change in each brain region and the time score showed an inverse correlation on the surgical side. In the future, it would appear to be necessary to increase the number of cases and consider non-linear multiple regression analysis, i.e., machine learning. 
Table 3 Stepwise multiple linear regression analysis predicting time score change

\begin{tabular}{lcccccccc}
\hline & Estimate & Std. error & $t$ value & $\operatorname{Pr}(>|\mathrm{t}|)$ & CI & Standard $\beta$ & Logworth & VIF \\
\hline (Intercept) & 1.2425177 & 0.660593 & 1.88 & 0.0894 & -0.229375 to 2.71441 & 0 & \\
CVR change in Rt uncus & -0.016778 & 0.003943 & -4.25 & 0.0017 & -0.025565 to -0.007992 & -1.10135 & 2.775 & 2.839741 \\
CVR change in Rt middle occipital & 0.0274737 & 0.009141 & 3.01 & 0.0132 & 0.0071053 to 0.047842 & 1.222319 & 1.879 & 7.0103066 \\
$\quad$ gyrus & & & & & & & \\
CVR change in Rt paracentral lobule & -0.01637 & 0.006567 & -2.49 & 0.0318 & -0.031001 to -0.001738 & -0.77574 & 1.497 & 4.1043386 \\
CVR change in Lt angular gyrus & 0.007726 & 0.003939 & 1.96 & 0.0782 & -0.00105 to 0.0165021 & 0.39835 & 1.107 & 1.7479002 \\
Age & -0.017928 & 0.009745 & -1.84 & 0.0957 & -0.039641 to 0.0037857 & -0.40824 & 1.019 & 2.0870254 \\
Sex [F] & 0.114909 & 0.081888 & 1.4 & 0.1908 & -0.067549 to 0.297367 & 0.242789 & 0.0719 & 1.2687193 \\
CVR change in Rt gyrus rectus & 0.0008638 & 0.004467 & 0.19 & 0.8505 & -0.009089 to 0.0108163 & 0.053486 & 0.07 & 3.2417151 \\
\hline
\end{tabular}

Variables entered into the model were age, sex, laterality of EC-IC bypass, and CVR change in Lt middle frontal gyrus, Rt gyrus rectus, Lt paracentral lobule, Rt paracentral lobule, Lt inferior parietal lobule, Lt angular gyrus, Lt postcentral gyrus, Rt middle occipital gyrus, Lt anterior cingulate, and Rt uncus. The table shows the final step of the analysis. The adjusted coefficient of determination $\left(R^{2}\right)$ calculated by leave-onesubject-out cross-validation was 0.1391 . The VIF of each individual variable was $<3.5$, indicating minimal collinearity

$\beta$ standard partial regression coefficient, $C I$ 95\% confidence interval for each regression coefficient, VIF variance inflation factor

Table 4 Stepwise multiple linear regression analysis predicting accuracy score change

\begin{tabular}{|c|c|c|c|c|c|c|c|c|}
\hline & Estimate & Std. error & $t$ value & $\operatorname{Pr}(>|t|)$ & $\mathrm{CI}$ & Standard $\beta$ & Logworth & VIF \\
\hline (Intercept) & -0.003299 & 0.004766 & -0.69 & 0.5009 & -0.013594 to 0.0069962 & 0 & & \\
\hline CVR change in Rt cingulate gyrus & -0.000876 & 0.000323 & -2.71 & 0.0177 & -0.001573 to -0.000179 & -0.68727 & 1.752 & 2.4578475 \\
\hline CVR change in Rt precuneus & 0.0008368 & 0.000345 & 2.43 & 0.0304 & 0.000092254 to 0.0015813 & 0.554067 & 1.517 & 1.9969942 \\
\hline CVR change in Lt precentral gyrus & 0.0004376 & 0.000221 & 1.98 & 0.0698 & -0.00004089 to 0.0009161 & 0.444929 & 1.156 & 1.9448464 \\
\hline $\begin{array}{l}\text { CVR change in Rt superior occipi- } \\
\text { tal gyrus }\end{array}$ & 0.0004706 & 0.000256 & 1.84 & 0.0893 & -0.00008316 to 0.0010243 & 0.421314 & 1.049 & 2.019667 \\
\hline
\end{tabular}

Variables entered into the model were age, sex, laterality of EC-IC bypass, and CVR change in Lt precentral gyrus, Rt superior parietal lobule, Rt precuneus, bilateral superior occipital gyrus, Rt fusiform gyrus, bilateral thalamus, Rt cingulate gyrus, Rt parahippocampal gyrus, and Rt uncus. The table shows the final step of the analysis. Adjusted coefficient of determination $\left(R^{2}\right)$ calculated by leave-one-subject-out cross-validation was 0.2533 . The VIF of each individual variable was $<2.5$, indicating minimal collinearity

$\beta$ standard partial regression coefficient, $C I 95 \%$ confidence interval for each regression coefficient, VIF variance inflation factor

\section{Conclusions}

This study suggested the following. In hemodynamic ischemia (both CBF and CVR reduced), processing speed might be adjusted so that accuracy would not be lowered by the speed-accuracy trade-off mechanism. More data will be needed before the clinical implications of these findings become clear. However, we have pointed out the possibility for the first time that it is necessary to pay attention to the "speed-accuracy trade-off" for each brain region when investigating the effect of EC-IC bypass on cognitive function. Therefore, considering the results of the present study, a different concept in the discussion of the relationship between cerebral hemodynamics and cognitive function is proposed. Future studies may use speed-accuracy imbalance as one of the diagnostic methods for stage 2 hemodynamic ischemia. In that case, it may be possible to consider surgical indications for bypass surgery in daily medical practice without special equipment.
Supplementary Information The online version contains supplementary material available at https://doi.org/10.1007/s10143-021-01720-0.

Acknowledgements The authors would like to thank the SPECT and rehabilitation team at Asa City Hiroshima Citizens' Hospital for their effort.

Author contribution $\mathrm{KS}$ and $\mathrm{SH}$ conceived and designed the study. $\mathrm{SH}, \mathrm{AF}$, and TT performed the statistical analysis and interpreted the results. AY participated in data collection. AK, HA, SY, and TM interpreted the results. All authors reviewed and edited the manuscript.

Funding This work was supported by JST A-STEP Grant Number JPMJTM20RA, Japan.

Data availability The datasets generated and/or analyzed in the current study are available from the corresponding author upon reasonable request.

Code availability Not applicable. 


\section{Declarations}

Ethics approval All procedures performed in studies involving human participants were in accordance with the ethical standards of the institutional review boards and the 1964 Helsinki Declaration and its later amendments or comparable ethical standards. This retrospective study was approved by the Research Ethics Committee of Hiroshima City Asa Citizens Hospital and Hiroshima University (E-466-3, E-1554-2).

Consent to participate Written, informed consent was obtained from all individual participants included in the study.

Consent for publication Not applicable.

Competing interests Co-authors Akihiko Kandori and Hirokazu Atsumori are employed by Hitachi Ltd., Tokyo, Japan. The remaining authors declare that they have no conflict of interest.

Open Access This article is licensed under a Creative Commons Attribution 4.0 International License, which permits use, sharing, adaptation, distribution and reproduction in any medium or format, as long as you give appropriate credit to the original author(s) and the source, provide a link to the Creative Commons licence, and indicate if changes were made. The images or other third party material in this article are included in the article's Creative Commons licence, unless indicated otherwise in a credit line to the material. If material is not included in the article's Creative Commons licence and your intended use is not permitted by statutory regulation or exceeds the permitted use, you will need to obtain permission directly from the copyright holder. To view a copy of this licence, visit http://creativecommons.org/licenses/by/4.0/.

\section{References}

1. Derdeyn CP, Grubb RL Jr, Powers WJ (1999) Cerebral hemodynamic impairment: methods of measurement and association with stroke risk. Review Neurology 53(2):251-259. https://doi.org/10. 1212/wnl.53.2.251

2. Fierstra J, van Niftrik C, Warnock G, Wegener S, Piccirelli M, Pangalu A, Esposito G, Valavanis A, Buck A, Luft A, Bozinov O, Regli L (2018) Staging hemodynamic failure with blood oxygenlevel-dependent functional magnetic resonance imaging cerebrovascular reactivity: a comparison versus gold standard ((15)O-) $\mathrm{H}(2) \mathrm{O}$-positron emission tomography. Stroke 49(3):621-629. https://doi.org/10.1161/STROKEAHA.117.020010

3. Fisher JA, Venkatraghavan L, Mikulis DJ (2018) Magnetic resonance imaging-based cerebrovascular reactivity and hemodynamic reserve. Stroke 49(8):2011-2018. https://doi.org/10.1161/ STROKEAHA.118.021012

4. Grubb RL Jr, Derdeyn CP, Fritsch SM, Carpenter DA, Yundt KD, Videen TO, Spitznagel EL, Powers WJ (1998) Importance of hemodynamic factors in the prognosis of symptomatic carotid occlusion. JAMA 280(12):1055-1560. https://doi.org/10.1001/ jama.280.12.1055

5. JET Study Group (2002) Japanese EC-IC bypass trial (JET study): the second interim analysis. Surg Cereb Stroke 30:434-437. https://doi.org/10.2335/scs.30.434 (Japanese)

6. Kataoka H, Miyamoto S, Ogasawara K, Iihara K, Takahashi JC, Nakagawara J et al (2015) Results of prospective cohort study on symptomatic cerebrovascular occlusive disease showing mild hemodynamic compromise [Japanese Extracranial-Intracranial Bypass Trial (JET)-2 Study]. Neurol Med Chir (Tokyo) 55:460 468. https://doi.org/10.2176/nmc.oa.2014-0424
7. Ogasawara K, Ogawa A, Yoshimoto T (2002) Cerebrovascular reactivity to acetazolamide and outcome in patients with symptomatic internal carotid or middle cerebral artery occlusion: a xenon-133 single-photon emission computed tomography study. Stroke 33(7):1857-1862. https://doi.org/10.1161/01.str.00000 19511.81583.a8

8. Powers WJ, Clarke WR, Grubb RL Jr, Videen TO, Adams HP Jr, Derdeyn CP, Investigators COSS (2011) Extracranial-intracranial bypass surgery for stroke prevention in hemodynamic cerebral ischemia: the Carotid Occlusion Surgery Study randomized trial. JAMA 306(18):1983-1992. https://doi.org/10.1001/jama.2011. 1610

9. Fantini S, Sassaroli A, Tgavalekos KT, Kornbluth J (2016) Cerebral blood flow and autoregulation: current measurement techniques and prospects for noninvasive optical methods. Neurophotonics 3:031411. https://doi.org/10.1117/1.NPh.3.3.031411

10. Iadecola C (2013) The pathobiology of vascular dementia. Review Neuron 80(4):844-866. https://doi.org/10.1016/j.neuron.2013.10. 008

11. Ishikawa M, Kusaka G, Terao S, Nagai M, Tanaka Y, Naritaka $H$ (2017) Improvement of neurovascular function and cognitive impairment after STA-MCA anastomosis. J Neurol Sci 373:201207. https://doi.org/10.1016/j.jns.2016.12.065

12. Kolb B, Fadel H, Rajah G, Saber H, Luqman A, Rangel-Castilla L (2019) Effect of revascularization on cognitive outcomes in intracranial steno-occlusive disease: a systematic review. Neurosurg Focus 46:E14. https://doi.org/10.3171/2018.11.FOCUS 18517

13. Marshall RS, Festa JR, Cheung YK, Pavol MA, Derdeyn CP, Clarke WR, Videen TO, Grubb RL, Slane K, Powers WJ, Lazar RM, Investigators RECON (2014) Randomized Evaluation of Carotid Occlusion and Neurocognition (RECON) trial: main results. Neurology 82(9):744-751. https://doi.org/10.1212/WNL. 0000000000000167

14 Brandstater ME (2005) Stroke rehabilitation. In: DeLisa JA, Gans BM (eds) Physical medicine and rehabilitation: principles and practice, 4th edn. Lippinctt Williams \& Wilkins, Philadelphia, PA, pp 1655-1676

15. Murakami T, Hama S, Yamashita H, Onoda K, Hibino S, Sato H, Ogawa S, Yamawaki S, Kurisu K (2014) Neuroanatomic pathway associated with attentional deficits after stroke. Brain Res 1544:25-32. https://doi.org/10.1016/j.brainres.2013.11.029

16. Shimonaga K, Hama S, Tsuji T, Yoshimura K, Nishino S, Yanagawa A et al (2021) The right hemisphere is important for drivingrelated cognitive function after stroke. Neurosurg Rev 44:977985. https://doi.org/10.1007/s10143-020-01272-9

17. Skurvydas A, Juodzbaliene V, Darbutas T, Brazaitis M (2018) One year after ischemic stroke: changes in leg movement path stability in a speed-accuracy task but no major effects on the hands. Hum Mov Sci 57:50-58. https://doi.org/10.1016/j.humov.2017.11.005

18. Dayan E, Cohen LG (2011) Neuroplasticity subserving motor skill learning. Neuron 72:443-454. https://doi.org/10.1016/j.neuron. 2011.10.008

19. Hoshi H, OhniShi T, Jinnouchi S, Futami S, Nagamachi S, Kodama T, Watanabe K, Ueda T, Wakisaka S (1994) Cerebral blood flow study in patients with moyamoya disease evaluated by IMP SPECT. J Nuci Med 35:44-50

20. Mizumura S, Kumita S, Cho K, Ishihara M, Nakajo H, Toba M, Kumazaki T (2003) Development of quantitative analysis method for stereotactic brain image: assessment of reduced accumulation in extent and severity using anatomical segmentation. Ann Nucl Med 17:289-295. https://doi.org/10.1007/BF02988523

21. Ishii K, Uemura T, Miyamoto N, Yoshikawa T, Yamaguchi T, Ashihara T, Ohtani Y (2011) Regional cerebral blood flow in healthy volunteers measured by the graph plot method with 
iodoamphetamine SPECT. Ann Nucl Med 25:255-260. https:// doi.org/10.1007/s12149-010-0451-1

22. Stine RA (1995) Graphical interpretation of variance inflation factors. Am Stat 49(1):53-56

23. Logan GD, Van Zandt T, Verbruggen F, Wagenmakers EJ (2014) On the ability to inhibit thought and action: general and special theories of an act of control. Psychol Rev 121:66-95. https://doi. org/10.1037/a0035230

24. Mallet N, Schmidt R, Leventhal D, Chen F, Amer N, Boraud T, Berke JD (2016) Arkypallidal cells send a stop signal to striatum. Neuron 89:308-316. https://doi.org/10.1016/j.neuron.2015.12.017

25. Schmidt R, Berke JD (2017) A pause-then-cancel model of stopping: evidence from basal ganglia neurophysiology. Philos Trans R Soc Lond B Biol Sci 372(1718). https://doi.org/10.1098/rstb. 2016.0202 .

26. Wang CT, Lee CT, Wang XJ, Lo CC (2013) Top-down modulation on perceptual decision with balanced inhibition through feedforward and feedback inhibitory neurons. PLoS One 8(4):e62379. https://doi.org/10.1371/journal.pone.0062379
27. Wilson KM, de Joux NR, Finkbeiner KM, Russell PN, Retzler JR, Helton WS (2018) Prolonging the response movement inhibits the feed-forward motor program in the sustained attention to response task. Acta Psychol (Amst) 183:75-84. https://doi.org/10.1016/j. actpsy.2018.01.001

28. Drewes J, Goren G, Zhu W, Elder JH (2016) Recurrent processing in the formation of shape percepts. J Neurosci 36:185-192. https:// doi.org/10.1523/JNEUROSCI.2347-15.2016

29. Shatzman S, Mahajan S, Sundararajan S (2016) Often overlooked but critical: poststroke cognitive impairment in right hemispheric ischemic stroke. Stroke 47:e221-223. https://doi.org/10.1161/ STROKEAHA.116.014280

Publisher's note Springer Nature remains neutral with regard to jurisdictional claims in published maps and institutional affiliations. 